Article

\title{
Emerging Technology and Business Model Innovation: The Case of Artificial Intelligence
}

\author{
Jaehun Lee ${ }^{1, *}$, Taewon Suh ${ }^{2}$, Daniel Roy ${ }^{2}$ and Melissa Baucus ${ }^{2}$ \\ 1 AI Center, Samsung Research, Seoul 05510, Korea \\ 2 McCoy College of Business, Texas State University, San Marcos, TX 78666, USA \\ * Correspondence: i.am.jaehun@gmail.com; Tel.: +82-10-5155-3425
}

Received: 6 June 2019; Accepted: 16 July 2019; Published: 22 July 2019

\begin{abstract}
Artificial intelligence (AI) has been altering industries as evidenced by Airbnb, Uber and other companies that have embraced its use to implement innovative new business models. Yet we may not fully understand how this emerging and rapidly advancing technology influences business model innovation. While many companies are being made vulnerable to new competitors equipped with AI technology, this study attempts to focus on the proactive side of the use of AI technology to drive business model innovation. Describing AI technology as the catalyst of business model innovation, this study sheds light on contingent factors shaping business model innovation initiated by the emerging technology. This study first provides a brief overview of AI, current issues being tackled in developing AI and explains how it transforms business models. Our case study of two companies that innovated their business models using AI shows its potential impact. We also discuss how executives can create an innovative AI-based culture, which rephrases the process of AI-based business model innovation. Companies that successfully capitalize on AI can create disruptive innovation through their new business models and processes, enabling them to potentially transform the global competitive landscape.
\end{abstract}

Keywords: business model innovation; artificial intelligence; case study; emerging technology

\section{Introduction}

Companies around the globe are seeing their industries disrupted by new technologies that result in business model innovation [1]. Artificial intelligence (AI) - "Intelligent systems created to use data, analysis and observations to perform certain tasks without needing to be programmed to do so" [2] - represents the most important technological development. AI disrupts industries and companies when companies use it to create innovative new business models [3]. Companies such as Amazon, Uber, Tesla, Google, Alibaba and UPS, along with many other companies have innovated their business models and enhanced their competitive advantages using AI. Top executives need to embrace an entrepreneurial and innovative mindset and instill this mindset using AI throughout their organizations to remain competitive and viable.

The concept of business model innovation has been put to the forefront of the debate of how companies may preserve their market position [4,5]. The present literature of business model innovation mainly focuses on external antecedents, which may pressure companies to engage in business model innovation [6]. This pressure may also arise through technological disruptions. Researchers argue that the process of business model innovation is prone to being affected by their environment [7]. That is, while the literature mainly focuses on external factors that may pressure companies to engage in business model innovation, one recently blossoming research stream examines how the introduction of new technology engages companies to innovate their business model [8,9]. Regretfully, however, studies investigating the direct impact of emerging technologies on business model innovation are 
few and far between. A few studies have only recently explored AI technology meticulously in consideration of business model innovation (e.g., [10,11]). For instance, Garbuio and Lin [10] identified seven emerging business model archetypes in their timely and critical analysis of AI-driven health care startups.

However, more studies need to be added to this particular topic area. The expected consequences of AI technology to business model innovation would be much more intense in the multifaceted elements of business model innovation. Therefore, endeavoring to generate a holistic view on how companies, particularly using AI technology, create value and how they perform the activities needed to do so, we attempt to discuss the plausible consequences of AI technology in business model innovation. It is noticeable that this study considers a business model to be an activity system or a set of interdependent activities spanning firm boundaries [12]. Thus, our case study on the human resource space may be able to shed light on further understanding the business model innovation initiated by AI technology.

The following section explains the types of AI, which are symbolic and neural AI. It also addresses two of the most important issues currently affecting the implementation of AI technology, namely, combining symbolic and neural AI and dealing with the quality and quantity of data in AI systems. Our case study in Section 3 exemplifies the use of AI technology for business model innovation, showing ways that AI can help managers tackle and rethink key organizational problems, altering their business model in the process. The brief overview of AI and the following case study provide the basis for our discussion in Section 4 in regard to how executives can create an innovative AI-based business model. We conclude our discussion in Section 5 with advice on how to manage the large-scale organizational change required to embrace business model innovation associated with the use of AI systems.

\section{AI Technology: Definitions and Trends}

The inspiration of artificial intelligence (AI) was to create an autonomous machine capable of human-like thinking [13]. In 1956, John McCarthy organized a research group and coined the concept of AI. The group assumed every aspect of learning or any other feature of intelligence can be so precisely described that it could be simulated by a machine. Applications include determining how to make machines use language, form abstractions and concepts, and solve complicated problems [14]. The massive and growing data available today and the steady improvements of computational power and algorithms have generated numerous applications of AI across many diverse industries. Although definitions and concepts of AI vary with the goals and domain, the main characteristic of AI is mimicking human cognitive function, particularly learning and problem solving. It is notable that the concept proposed in 1956 is considered still relevant to current AI research.

\subsection{Types of AI Technology Development Approaches: Symbolic vs. Neural}

Recently, the deep neural network known as deep learning has emerged as a dominant force among AI development approaches with outstanding performance in the fields of image/voice recognition, natural language processing and predictive techniques. However, deep neural networks are not suitable for solving all AI-related problems.

\subsubsection{Symbolic AI}

Before the era of deep learning from AlphaGo [15], symbolic AI had been the predominant paradigm among AI development approaches. It assumes that high-level representation of knowledge (symbol) and combinations of symbols can achieve human-like AI by performing reasoning in a manner similar to how humans express their thoughts and draw conclusions from deductive reasoning [16]. The example of deductive reasoning from symbolic AI is like the old Roman saying:

All men are mortal. Caius is a man. Therefore, Caius is mortal.

Symbolic AI includes any programming methods and systems that use symbols such as letters and numbers to encode a human's knowledge, rule-based operations, and determined policy. Symbolic AI 
can provide companies with competitive advantages by producing results that humans can interpret, predict and use quickly. Expert systems, although the degree of success they achieve as AI is controversial, represent the most well-known and widespread AI systems. Specifically, in the fields of manufacturing and production, many expert systems enable companies to automate time-consuming and knowledge-intensive tasks in various operations such as design, process planning, production control and diagnosis [17].

IBM's Deep Blue chess-playing computer that defeated the world champion in 1996 is one of the outstanding symbolic AI systems that exploits symbolic, rule-based knowledge [18]. More recently, Google has capitalized on symbolic AI systems to provide the most relevant and important information in the top box under the queries delivered via the Google search engine. Figure 1 shows the search result from the term, 'Seoul' by Google's Knowledge Graph. Google's Knowledge Graph illustrates an outstanding symbolic AI system, incorporating knowledge and reasoning methods to enhance search results. Note that the current knowledge graph contains more than 18 billion facts and 570 million entities [19]. Information from Knowledge Graph and reasoning technologies are used to produce answers in Google Search Engine, Google Assistant and Google Home voice queries [20].

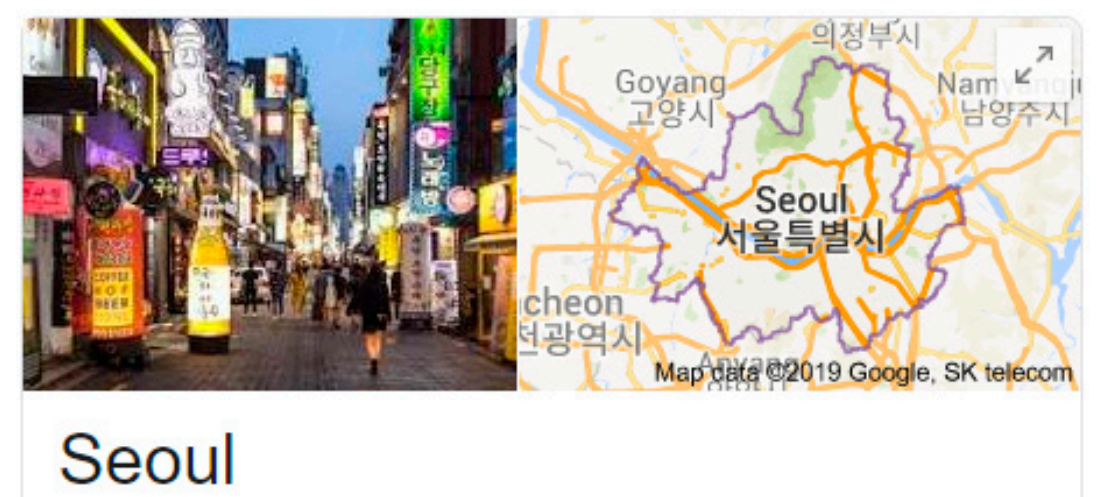

Capital of South Korea

Seoul, the capital of South Korea, is a huge metropolis where modern skyscrapers, high-tech subways and pop culture meet Buddhist temples, palaces and street markets. Notable attractions include futuristic Dongdaemun Design Plaza, a convention hall with curving architecture and a rooftop park; Gyeongbokgung Palace, which once had more than 7,000 rooms; and Jogyesa Temple, site of ancient locust and pine trees.

Area: $605.2 \mathrm{~km}^{2}$

Elevation: $38 \mathrm{~m}$

Weather: $6^{\circ} \mathrm{C}$, Wind NW at $2 \mathrm{~m} / \mathrm{s}, 37 \%$ Humidity

Local time: Sunday 1:53 PM

Population: 9.776 million (2017) United Nations

Figure 1. Search result by Google's Knowledge Graph.

Symbolic AI works best with static problems, but it has some stumbling blocks. It is labor intensive, challenging and expensive for developers to create symbolic AI systems as they depend on gathering and understanding complicated and implicit expertise and knowledge. Additionally, symbolic AI is not suitable in situations requiring recognition, since it is difficult to represent recognition knowledge efficiently. 


\subsubsection{Neural AI}

A huge part of AI's explosive growth has been made possible with the contribution of machine learning. Technically, machine learning approaches involve using algorithms to improve learning performance on a specific task by relying on patterns generated from practice or sample data. Neural AI relies on an artificial neural network (ANN) or an aggregate of machine learning algorithms that work in sync to mimic the human brain to solve more complex problems and learn in a manner similar to neurons in a human brain. It enables computers to learn from data without being given explicit knowledge. Technically, machine learning approaches can be classified into three areas: (1) supervised learning, that involves learning from correct answers (labeled data); (2) unsupervised learning, defined as finding knowledge or information when given some raw data (unlabeled data); and (3) reinforcement learning that entails how agents in an environment take action to maximize their rewards.

The details on artificial neural networks can be found in [21]. Recently, deep neural networks have been receiving a lot of attention and application in industry, as well as in academia. As a part of machine learning, it is based on deeper layers of the ANN that allows it to solve more complicated problems and to learn from raw data without much hand-based preprocessing of data. It has shown outstanding performance on speech/image recognition, fraud detection, providing recommendations, and natural language processing. Thus, leading technology companies such as Google and Facebook have announced innovative developments based on deep learning-based speech/image recognition [22,23]. Netflix, Spotify and Amazon use machine learning algorithms to generate personalized recommendations [24,25]. Neural AI has been successful in dealing with well-defined problems such as recognition and perception with a lot of labeled data. At the current stage of development, neural AI could be criticized since it is far from human-like thinking or reasoning and it needs a lot of labeled data $[26,27]$.

\subsection{The Current Trends in AI Technology Development}

Experts agree that $\mathrm{AI}$ is still in the early days. Much work remains to reach a level of efficiency that allows for scaling learning and reasoning capabilities across broader applications. In this regard, Yoshua Bengio claimed that AI research needs to extend the capability for reasoning, learning causality, and exploring the world in order to learn and acquire information [28]. Approaches that combine symbolic AI with neural AI appear promising for addressing these issues.

\subsubsection{Marrying Symbolic AI and Neural AI}

Symbolic AI joined with neural AI would help address the fundamental challenges of human-like reasoning, knowledge representation, learning with lack of data or transfer learning, and interpretability to resolve the black-box issue [29], which is associated with unknown internal structure of AI to lower the degree of the AI's transparency as well as the extent to which the AI is supervised by humans [29]. Studies related to this are not limited to the area of computer science, but extend to cognitive science, neural science, philosophy, psychology, and several other areas. A few notable studies could be found in [30-33]. Specifically, the MIT-IBM Watson AI Lab launched a project titled, 'Empowering AI with symbolic reasoning', to perform more complicated tasks [30]. Garcez et al. proposed a novel method for neural symbolic integration [31], and they verified the feasibility of their proposed method by implementing logic tensor networks [32]. Pedro Domingos is leveraging a combination of symbolic AI and neural AI in the machine reading area [33]. Marta Garnelo developed an end-to-end reinforcement learning architecture comprising a neural back-end and a symbolic frond-end with the potential to address the challenges [34]. These examples illustrate various ways that researchers are progressing in developing solutions that marry symbolic and neural AI. 


\subsubsection{Addressing Data Quality and Quantity Issues}

'Garbage-in, garbage-out' is a basic principle for AI. To learn or reason properly, historical data should meet the standards of high-quality and sufficient quantity. Unfortunately, most data do not meet these standards; thus, AI researchers and data scientists have no choice but to use 80 percent of their time on data preprocessing to ensure high quality. In order to address the data quality issue, leading AI startups have been developing tools to automate data preprocessing. Paxata and Trifacta have developed a cloud-based solution for ensuring data quality and enrichment [35,36], whereas, Tamr and Alation created a method and solution for addressing data silo issues [37,38].

Data quantity issues are also being solved by combining symbolic AI and neural AI. This can ensure performance without the volume of data since symbolic AI does not require as much data as neural AI. Additionally, Zoubin Ghahramani proposed a probabilistic machine learning method to resolve data quantity issue [39]; this method addresses more issues than data quantity, because it uses a new machine learning paradigm to resolve data uncertainty.

\section{Case Study: Need for AI to Innovate Business Model}

\subsection{Case \#1: A Bigger Manufacturing Company}

We use an exploratory case study with the aim to understand how the phenomenon of AI-based business model innovation takes place. A case study is a qualitative research method that allows an in-depth investigation of a contemporary phenomenon within its real-life context. Case studies attempt to understand the dynamics present with single settings by using a variety of lenses to reveal multiple facets of the phenomenon [40,41].

This section presents an example case in a large manufacturing company (Company A, hereafter) where the introduction and use of AI and analytics led to business model innovation, specifically in the area of human resources or talent management. Note that this case is illustrating a machine learning approach involving using algorithms to improve learning performance on a specific task. In other words, this example case is associated with the application of a type of neural AI, deep neural network, the type of $\mathrm{AI}$ in which we learn from data to solve more complicated problems without much hand-based preprocessing of the data.

Company A was struggling to add over 600 production employees. The labor market was exceptionally tight, and efforts to attract and hire talent, which had not changed in many years, were proving ineffective. Market conditions, tools and resources relating to attracting and retaining talent had changed dramatically, but Company A's approach had not; placing it at a significant competitive disadvantage. Company executives were unaware of the dramatic changes that had taken place in the talent management space but did recognize they lacked the internal expertise to innovate to solve the people related challenges. Company A's executive leadership reached out to an external company with expertise in the talent management space. The external company was known for its innovative approaches including the use of AI to improve Human Resource processes and performance.

Historically, the company's efforts to attract and hire talent focuses on finding candidates with three to five years of prior experience in the manufacturing area. When proprietary technology (AI and algorithms) was used to analyze years of employee related data, which included prior work experience, educational attainment, job-related performance evaluations, and turnover-it produced surprising results suggesting that many of the company's best performing production employees had little to no prior manufacturing experience. The vast majority, however, had at least three consecutive (uninterrupted) years of experience working in fast food.

Based upon the results, the company altered its recruiting strategies focusing instead on identifying and reaching high performing employees working in the fast food industry. It is considerably easier to find fast food workers than it is to find people with manufacturing experience. The company was able to achieve its hiring objectives, reduce turnover and lower its cost of employee acquisition. The company now uses a fully integrated approach to managing the employee lifecycle using advanced 
software with AI to strengthen organizational alignment ensuring the people strategies are effective and fully aligned with the organizational objectives.

The use of AI, in this case a simple algorithm, enabled the company to quickly hone in on the human resources necessary for growing the company and improving productivity. This case illustrates that deep learning from data can produce innovative solutions that create value.

\subsection{Case \#2: A Ready-Mix Concrete Company}

We present an additional case of a company to cross-sectionally understand how business models can be innovated through AI technology. This case involves a sizeable family-owned company (Company B, hereafter) that produces ready-mix concrete, with over 30 locations across Texas. Company $\mathrm{B}$ hires people with a commercial driver license (CDL).

In this case, the business owner had trucks sitting idle and idle trucks do not generate revenue. A key operational metric at Company B involves making sure every truck is being utilized daily; this metric revealed a people problem. The shortage of commercial drivers represents a market condition resulting from structural and non-structural factors [42], some of which are external to Company B but the company can influence internal factors such as recruiting strategies, pay, signing bonuses, work environment and so on.

New technologies have compressed the hiring cycle. In an exceptionally tight labor market, for example, the speed with which a company can move to attract and hire talent becomes increasingly important. Company B was responding too slowly, relying on strategies, systems and processes that in many cases had remained unchanged for decades. Paper applications simply cannot compete with the ability to apply almost instantly using your phone. These days qualified talent is snapped up with incredible speed. A reliance on traditional systems placed Company B at a tremendous disadvantage in the labor market, challenging their ability to find new drivers quickly. Management realized that their company's current systems were not enough, and most importantly, could not scale or deliver the speed required.

The same external company that worked for Company A with expertise in the talent management space was also invited to solve the problem. The external company examined Company B's current perceptions and processes with an emphasis on how technological advances and new business models (innovations) might be applied. Senior management was aware of the importance of the issue but not aware of the modern tools available to address it. After receiving inputs from outside expertise, they began to perceive the opportunities and importance of business model innovation using AI technology. However, they also agreed that the implementation and exploitation of new business model based on a new technology remain challenging given the Company's deliberately conservative culture which was reluctant to change.

As in many industries, the talent management space has evolved dramatically and rapidly. In the past, most of recruiting spending went towards classified ads until pioneering companies like Monster and CareerBuilder used technology to innovate their business models in a way that all but wiped out the use of classified ads as a recruiting tool. Indeed and other companies then began aggregating job postings, crawling the internet to find postings from multiple job boards and displaying them on a single site, a simple but powerful new approach. Companies like Ziprecruiter blended job postings with a more robust applicant tracking system, thus adding powerful analytics and machine learning to facilitate the hiring process. Advanced recruiting systems now tie directly into comprehensive information management systems, allowing companies to capture data throughout the employee life cycle and relate it directly to financial performance.

These new approaches open new possibilities for Company B, driving not only efficiency, but providing data that can connect a company's human resource strategies with its profitability strategies. The employee life cycle took on new meaning when systems captured data and the data were used strategically to influence outcomes in a positive way. When companies are laser-focused on their own core business, ready-mix concrete in this case, they may have a hard time recognizing how 
new technologies in recruiting and hiring have changed and how they might gain advantages from effectively adapting to these changes.

In the human resource space, the most important questions have traditionally been very difficult to answer. These include questions such as: who are the company's best employees; how do we identify them; where and how did we find them; what motivates these employees; what investments in training and development best increase their performance and value to the company; how long, on average, do these employees remain with the company; why do they leave; and how does their compensation relate to productivity. When Company B answered these questions utilizing solutions based on AI technology, they could propel the innovation of their business model, which allowed them to exploit new opportunity [43]. As known in the literature, business model innovation also ensures sustainable, competitive advantages [44], facilitates adaptive changes in the environment [45], and enables companies to stay competitive in new environmental settings [46].

\section{Developing AI-Based Business Model}

Business model denotes an activity system or a set of interdependent activities spanning firm boundaries [12], and business model innovation is defined as a significant change in the company's operations and value creation, typically resulting in an improvement in firm performance [12,47]. AI has been fostering business model innovation across industries including technology/media, consumer products, financial services, health care, industrial, energy, public sector, and so on [32]. Interviews with more than 3,000 business executives revealed that 84 percent think AI will enable their companies to obtain or sustain a competitive advantage, and 75 percent state that AI will allow them to move into new businesses and ventures [48]. In this context, what should we consider to develop or innovate our business model with AI?

Andrew $\mathrm{Ng}$ released an AI playbook to transform companies with AI by drawing insights from his experiences leading Google Brain and Baidu AI [49]. The five steps outlined in [49] are discussed below, along with issues that we believe must be raised for each step. The deliberation in this section further illustrates the process of business model innovation in the actual cases in the previous section. Figure 2 summarizes the discussion and issues.

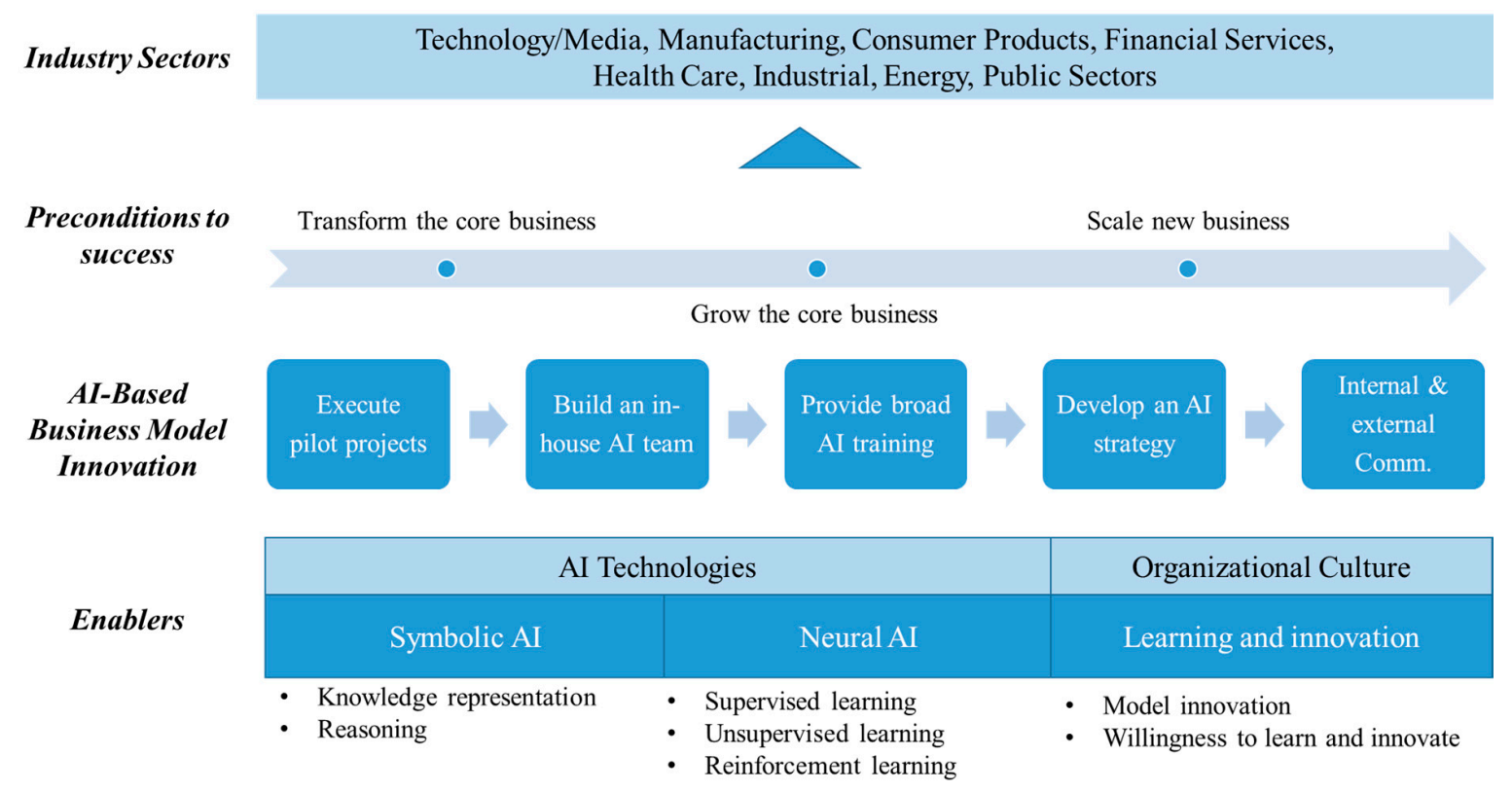

Figure 2. Developing an Artificial intelligence (AI)-Based Business Model. 


\subsection{Execute Pilot Projects to Gain Momentum}

Early in the use of AI, a company needs to have pilot projects that succeed in order to build momentum toward business model innovations. Successful small-scale pilot projects allow employees to become familiar with AI technology, recognize that it does not mean the loss of their jobs, and generate enthusiasm for the use of AI. Commonly, many companies are so soaked in the AI syndrome that they fail to achieve their technical goals. Companies should instead focus on technically feasible smaller projects that are readily achievable. The adoption of AI represents and should be managed as a major organizational change effort [50], including making information that explains the why and how of the pilot projects available to all employees in understandable language. Furthermore, in designing a team for the projects, AI experts should be mingled with domain-experts such as human resource managers, marketing or social media experts, or employees well-versed in operations. It is obvious that the knowledge from domain experts is so essential for symbolic AI and helpful for neural AI and these experts can offer diverse perspectives on the problem being tackled.

\subsection{Build an in-House AI Team}

Andrew Ng recommended building an in-house AI team to execute projects efficiently [49]. This is natural if companies want to build a more unique competitive advantage or have tremendous confidential data such as a customer usage log. Small and medium sized companies or new startups often cannot afford to hire a significant number of AI researchers and data scientists so they will need to consider alternative strategies. They may need to out-source AI to another company or form a joint venture with an AI company to get the necessary expertise. This reliance on "outside" experts will have to be managed carefully so competitors do not gain access to the company's activities.

\subsection{Provide Broad AI Training}

Most companies do not have enough AI researchers and experts, and companies find it difficult to hire them due to a shortage in the AI field. Thus, Andrew Ng suggested educating employees-all the way from training business executives down to AI researchers by utilizing digital content such as MOOCs [49]. Digital content is relatively affordable and allows a more personalized experience so it could be applied in small and medium sized companies. Companies may want to help develop additional content for AI education since it not only solves the current issue-the insufficiency of AI researchers-but it also fuels lasting AI business model innovation.

\subsection{Develop an AI Strategy}

The key to an AI strategy is to create the virtuous cycle of AI shown in Figure 3. For instance, Google has tremendous data, so it can build an accurate search engine as a product (A). This product enables Google to acquire more users (B), who then create more data on Google (C).

The key factor in AI is to have good quality and sufficient quantity of data. Companies too often attempt to drive AI without appropriate data like building a palace on quicksand. Data acquisition and data infrastructure are vital to transforming the business model. Approaches such as the lean startup method [51] encourage companies to develop minimum viable products that they get customers to use, gathering data in order to test which designs and features are most viable: this virtuous cycle is essential for building an innovative business model and is used in a variety of contexts including new venture startups. Companies using the virtuous cycle will recognize that building a good platform (or new business model) becomes an open-ended challenge. 


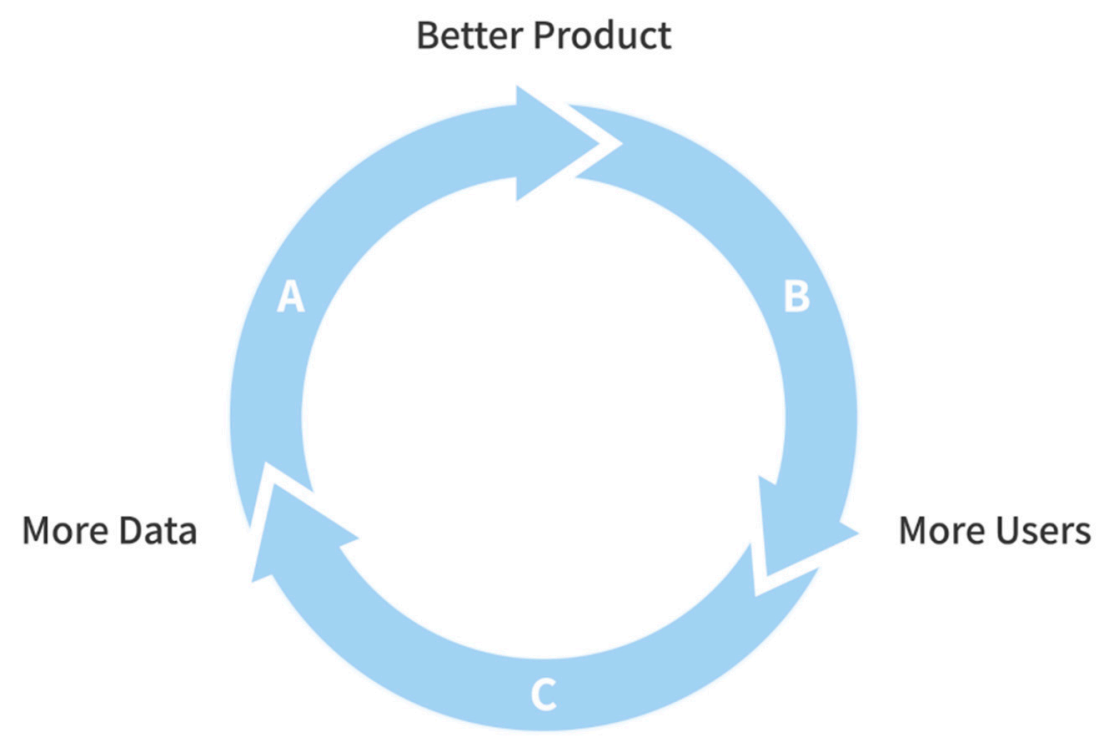

Figure 3. The virtuous cycle of AI.

\subsection{Develop Internal and External Communications}

The company's stakeholders need to be informed about how AI is transforming the business model and the consequences this has for them. Protecting the privacy of customers' and employees' data has become a major issue for companies, as well as ensuring that their actions and decisions comply with laws, regulations and ethical standards, and use of AI to process data and make decisions will create bigger challenges in these areas. Business model innovations developed through the use of AI will increase the company's value to stockholders and provide opportunities to enhance value for customers. Innovations often arise from communications with a company's customers who suggest ideas and highlight what they do not like about current offerings and processes [52] so two-way communication may lead to greater business model innovations. Since AI is not well understood and AI technologies are changing very rapidly, companies will need to inform and educate all of their stakeholders as to how they use AI, the benefits of its use and potential drawbacks or limitations of AI.

\section{Discussion and Conclusions}

The current study contributes to academia and practice in two ways. First, this study attempted to describe AI technology as the catalyst of business model innovation. More studies are called for to associate emerging technologies to business model innovation. Second, this study sheds light on contingent factors shaping business model innovation initiated by the emerging technology. Our case study and subsequent discussion on the creation of AI-based business model innovation provide insights on such contingent factors. However, for future study, more of those factors need to be fully listed and quantitatively tested.

Advances in AI technology and data analytics will continue to create opportunities and challenge delivery systems. Effective leaders need to find new and innovative ways to leverage these advances to transform their organization and drive growth. Many leaders will find that these advances take them in directions they had never considered. The focus on a company's core competencies and business strategy remains imperative but remaining open to and encouraging innovations that shift the company's business model represents a major challenge for leaders.

According to KPMG's 2017 CEO Survey, nearly 60 percent of leaders indicated their organizations lack the sensory and innovative processes to respond to rapid disruption. It follows that innovation has become a key focus of business leaders, but companies struggle to position themselves to detect emerging signals of disruption and to respond. This is particularly true for companies that have been in business for a number of years and achieved remarkable success, i.e., legacy businesses. Accenture 
recently published an article, "Make Your Wise Pivot to the New." In it, they discuss the fact that "C-level executives know that clinging to their legacy business can undermine their company's future health." The authors go on to suggest that three preconditions pave the way to success: transform the core business, grow the core business, and scale new business. We believe that for many companies, transforming and growing the core business will take priority over scaling a new business since companies must focus most of their attention on the business generating the most revenues. If companies start new businesses, these will likely be a byproduct of attempts to innovate around legacy businesses or perhaps more likely, legacy systems. Companies are increasingly looking for employees who can innovate and who have an entrepreneurial mindset because they recognize the need for employees who see major problems, view them as opportunities and implement innovative solutions.

In our ready-mix example, Company B had some of the most advanced technology in its trucks, allowing the drivers to deliver product more efficiently and thus more profitably; yet Company B had some of the most antiquated systems for managing the people side of its business. Without the people, the trucks sat idle. Only when the people side of the business began to affect the delivery side did they seek outside expertise to drive innovation through their talent management processes. Likewise, only when Company A needed to ramp to 800 employees, did it become hyper-focused on the people side of its business and seek knowledge and expertise it did not have. Applying data analytics to its people processes produced dramatic results, but it took an outside perspective and technological resources to achieve it.

It would be natural to ask why the two companies in our cases were unable to innovate internally to apply the latest advances in technology and update antiquated and ineffective systems. In part, the answer is that as with many companies today, these companies do not have AI teams on hand and have not considered how to effectively use AI according to the five recommendations we discussed earlier. If companies foster greater learning about AI and use of AI within their operations, this will help drive innovations across their companies.

Additionally, the culture of the organization likely plays a major role as the previous section of developing AI-base business model connotes. The literature suggests that organizational factors play a critical role in shaping the process of business model innovation [6]. The debate frequently revolves around contextual factors such as organizational design, organizational culture, and organizational values [6].

It seems developing a culture of innovation often runs contrary to an intentionally conservative and constrained view such as that expressed by the founder of the ready-mix company who indicated that he prefers to stay laser focus on core activities. Significant pivots require a certain degree of freedom to take risks and exposure to a lens or frame that is unfamiliar. Top managers play a key role in establishing the culture of an organization so they must model innovation and a willingness to continually learn and innovate. They could begin by learning about AI and how it can enhance the company's business model and systems, as well as encouraging and rewarding employees who acquire AI expertise and launch pilot projects in the organization.

This study has its limitations and thus calls for future study. The main limitation was the small number of cases in our case study. Although we conducted in-depth investigation and close observation to reveal both general and specific factors of the issues, the idiosyncratic conditions of different industries should be considered for the generalization of our findings. Future study also needs to exemplify different types of emerging business model archetypes. Different delivery models such as platform, SaaS (Software as a Service), and PaaS (Platform as a Service) can be discussed [10,53] in the context of AI-based business model innovation. Quantitative empirical studies are subsequently recommended, taking more organizations into account and providing another dimension of insights. Various methodological techniques can be utilized: e.g., Survey method will be able to introduce the detailed perceptions of industry leaders regarding the role of AI technology on business model innovation. Lastly, being absent in our study, ethical issues and challenges around a potential 
contradiction between rule-driven AI rationality and innovation uncertainty [54] should not be ignored in future study.

Author Contributions: Investigation, writing (original draft), J.L.; design, supervision, writing (original draft), writing (review \& editing), investigation, writing (original draft), writing (review \& editing), T.S. and D.R.; writing (review \& editing), M.B.

Funding: This Research received no external funding.

Conflicts of Interest: The authors declare no conflict of interest.

\section{References}

1. Wåge, D.; Crawford, G.E. Innovation in digital business models. In Proceedings of the 2017 XXVIII ISPIM Innovation Conference on Composing the Innovation Symphony, Vienna, Austria, 18-21 June 2017.

2. Antonescu, M. Are business leaders prepared to handle the upcoming revolution in business artificial intelligence? Qual. Access Success 2018, 19, 15-19.

3. Boitnott, J. 7 innovative companies using A.I. to distrust their industries. Available online: https:// www.inc.com/john-boitnott/7-innovative-companies-using-ai-to-disrupt-their-industries.html (accessed on 1 June 2019).

4. Sosna, M.; Trevinyo-Rodríguez, R.N.; Velamuri, S.R. Business model innovation through trial-and-error learning: The Naturhouse case. Long Range Plan. 2010, 43, 383-407. [CrossRef]

5. Wirtz, B.W.; Schilke, O.; Ullrich, S. Strategic development of business models: Implications of the Web 2.0 for creating value on the Internet. Long Range Plan. 2010, 43, 272-290. [CrossRef]

6. Foss, N.J.; Saebi, T. Fifteen years of research on business model innovation: How far have we come, and where should we go? J. Manag. 2017, 43, 200-227. [CrossRef]

7. Chesbrough, H. Business model innovation: Opportunities and barriers. Long Range Plan. 2010, 43, 354-363. [CrossRef]

8. Tongur, S.; Engwall, M. The business model dilemma of technology shifts. Technovation 2014, 34, 525-535. [CrossRef]

9. Velu, C. Business model innovation and third-party alliance on the survival of new firms. Technovation 2015, 35, 1-11. [CrossRef]

10. Garbuio, M.; Lin, N. Artificial intelligence as a growth engine for health care startups: Emerging business models. Calif. Manag. Rev. 2019, 61, 59-83. [CrossRef]

11. Valter, P.; Lindgren, P.; Prasad, R. Advanced business model innovation supported by artificial intelligence and deep learning. Wirel. Pers. Commun. 2018, 100, 97-111. [CrossRef]

12. Gassmann, O.; Frankenberger, K.; Sauer, R. A primer on theoretically exploring the field of business model innovation. The Eur. Bus. Rev. 2017, 4, 45-48.

13. McCorduck, P. Machines Who Think: A Personal Inquiry into the History and Prospects of Artificial Intelligence; AK Peters/CRC Press: Boca Raton, FL, USA, 2009.

14. McCarthy, J. A proposal for the Dartmouth summer research project on artificial intelligence. Ai Mag. 2006, $27,12$.

15. AlphaGo|Deepmind. Available online: https://deepmind.com/research/alphago/ (accessed on 15 May 2019).

16. Garcez, A.; D'Avila, S.; Lamb, L.C.; Gabbay, D.M. Neural-Symbolic Cognitive Reasoning; Springer Science \& Business Media: Berlin, Germany, 2008.

17. Iwata, K. Application of expert systems to manufacturing in Japan. Int. J. Adv. Manuf. Technol. 1988, 3, 23-37. [CrossRef]

18. Hsu, F. IBM's deep blue chess grandmaster chips. IEEE Micro 1999, 19, 70-81.

19. Newton, C. Google's Knowledge Graph Tripled in Size in Seven Months; CNET; CBS Interactive: San Francisco, CA, USA, 2017.

20. Bohn, D. Google Home: A Speaker to Finally Take on the Amazon Echo; The Verge; Vox Media: New York, NY, USA, 2017.

21. Schalkoff, R.J. Artificial Neural Networks; McGraw-Hill: New York, NY, USA, 1997; Volume 1. 
22. Chiu, C.; Sainath, T.N.; Wu, Y.; Prabhavalkar, R.; Nguyen, P.; Chen, Z.; Kannan, A.; Weiss, R.J.; Rao, K.; Gonina, E.; et al. State-of-the-art speech recognition with sequence-to-sequence models. In Proceedings of the 2018 IEEE International Conference on Acoustics, Speech and Signal Processing (ICASSP), Calgary, AB, Canada, 15-20 April 2018.

23. Taigman, Y.; Yang, M.; Ranzato, M.A.; Wolf, L. Deepface: Closing the gap to human-level performance in face verification. In Proceedings of the IEEE Conference on Computer Vision and Pattern Recognition, Columbus, OH, USA, 23-28 June 2014.

24. Gomez-Uribe, C.A.; Hunt, N. The Netflix recommender system: Algorithms, business value, and innovation. ACM Trans. Manag. Inf. Syst. 2016, 6, 13. [CrossRef]

25. Smith, B.; Linden, G. Two decades of recommender systems at Amazon.com. IEEE Internet Comput. 2017, 21, 12-18. [CrossRef]

26. What's Next for AI-Innovation Equation. Available online: https://www.ibm.com/watson/advantagereports/future-of-artificial-intelligence/ai-innovation-equation.html (accessed on 15 March 2019).

27. Marcus, G. Deep learning: A Critical Appraisal. Available online: https://arxiv.org/abs/1801.00631 (accessed on 15 March 2019).

28. One of the Fathers of AI is Worried About Its Future. Available online: https://www.technologyreview.com/ s/612434/one-of-the-fathers-of-ai-is-worried-about-its-future/ (accessed on 19 May 2019).

29. Besold, T.R.; Garcez, A.A.; Bader, S.; Bowman, H.; Domingos, P.; Hitzler, P.; Kuehnberger, K.-U.; Lamb, L.C.; Lowd, D.; Lima, P.M.V.; et al. Neural-Symbolic Learning and Reasoning: A Survey and Interpretation. Available online: https://arxiv.org/abs/1711.03902 (accessed on 19 May 2019).

30. Empowering AI with Symbolic Reasoning. Available online: https://mitibmwatsonailab.mit.edu/research/ empowering-ai-with-symbolic-reasoning/ (accessed on 19 March 2019).

31. Garcez, A.; Besold, T.R.; Raedt, L.; Foldiak, P.; Hitzler, P.; Icard, T.; Kuhnberger, K.-U.; Lamb, L.C.; Miikkulainen, R.; Silver, D.L. Neural-symbolic learning and reasoning: Contributions and challenges. In Proceedings of the AAAI Spring Symposium on Knowledge Representation and Reasoning: Integrating Symbolic and Neural Approaches, Palo Alto, CA, USA, 23-25 March 2015.

32. Serafini, L.; Garcez, A.A. Logic Tensor Networks: Deep Learning and Logical Reasoning from Data and Knowledge. Available online: http://ceur-ws.org/Vol-1768/NESY16_paper3.pdf (accessed on 19 March 2019).

33. Poon, H.; Domingos, P.M. Machine reading: A "killer app" for statistical relational AI. Stat. Relat. Artif. Intell. 2010, 10,6 .

34. Garnelo, M.; Arulkumaran, K.; Shanahan, M. Towards Deep Symbolic Reinforcement Learning. Available online: https://www.researchgate.net/publication/308320824_Towards_Deep_Symbolic_Reinforcement_ Learning (accessed on 19 March 2019).

35. Paxata. Available online: https://www.paxata.com/ (accessed on 21 March 2019).

36. Data Wrangling Tools and Software|Trifacta. Available online: https://www.trifacta.com/ (accessed on 21 March 2019).

37. Master Data Management System-Simplify Data Unification. Available online: https://www.tamr.com/ (accessed on 21 March 2019).

38. The Enterprise Data Catalog|Alation. Available online: https://alation.com/ (accessed on 21 March 2019).

39. Ghahramani, Z. Probabilistic machine learning and artificial intelligence. Nature 2015, 521, $452-459$. [CrossRef] [PubMed]

40. Stake, R.E. The Art of Case Study Research; Sage: Thousand Oaks, CA, USA, 1995.

41. Yin, R. Applications of Case Study Research; Sage Publications, Inc.: London, UK, 2003.

42. Lehmann, D.R. Market Research and Analysis; Irwin: Homewood, IL, USA, 1989; Volume 3.

43. Markides, C.C. Game-Changing Strategies: How to Create New Market Space in Established Industries by Breaking the Rules; John Wiley and Sons: Hoboken, NJ, USA, 2013.

44. Martins, L.L.; Rindova, V.P.; Greenbaum, B.E. Unlocking the hidden value of concepts: A Cognitive approach to business model innovation. Strateg. Entrep. J. 2016, 9, 99-117. [CrossRef]

45. Bouncken, R.B.; Fredrich, V. Good fences make good neighbors? Directions and safeguards in alliances on business model innovation. J. Bus. Res. 2016, 69, 5196-5202. [CrossRef]

46. Johnson, M.; Christensen, C.; Kagermann, H. Reinventing your business model. Harv. Bus. Rev. 2008, $86,50-59$. 
47. Boston Consulting Group. Business Model Innovation: When the Game Gets Tough, Change the Game; BCG: Boston, MA, USA, 2009.

48. How Artificial Intelligence is Revolutionizing Business in 2017. Available online: https://www.forbes.com/sites/ louiscolumbus/2017/09/10/how-artificial-intelligence-is-revolutionizing-business-in-2017/\#11ba98b15463 (accessed on 3 May 2019).

49. Ng, Y. AI Transformation Playbook. 2018. Available online: https://landing.ai/ai-transformation-playbook/ (accessed on 3 May 2019).

50. Palmer, I.; Dunford, R.; Buchanan, D. Managing Organizational Change; McGraw-Hill Education: New York, NY, USA, 2016.

51. Ries, E. The Lean Startup: How Today's Entrepreneurs Use Continuous Innovation to Create Radically Successful Businesses; Crown Business: New York, NY, USA, 2011.

52. Kavilanz, P. This Startup is Making Glasses Kids will Actually Want to Wear. 2019. Available online: https: //www.cnn.com/2019/01/23/success/pair-eyewear-childrens-glasses/index.html (accessed on 3 May 2019).

53. Duft, G.; Siekelova, A.; Kolencik, J. Incorporating cognitive artificial intelligence systems and real-time data analytics in clinical care delivery. Am. J. Med. Res. 2019, 6, 61-66.

54. Obschonka, M.; Audretsch, D.B. Artificial intelligence and big data in entrepreneurship: A new era has begun. Small Bus. Econ. 2019, 1-11. [CrossRef]

(C) 2019 by the authors. Licensee MDPI, Basel, Switzerland. This article is an open access article distributed under the terms and conditions of the Creative Commons Attribution (CC BY) license (http://creativecommons.org/licenses/by/4.0/). 